\title{
DEVELOPMENT OF MODELS FOR RECOGNITION OF TECHNOLOGICAL SITUATIONS IN THE OPERATION OF ELECTRIC CENTRIFUGAL PUMPS FOR OIL PRODUCTION
}

\author{
Vladimir Victorovich Bukhtoyarov*, Vadim Sergeevich Tynchenko, Eduard Arkadievich Petrovskiy, \\ Fedor Anatolyevich Buryukin \\ Siberian Federal University, Krasnoyarsk, Russia
}

The article deals with the problem of constructing models for automation of the technological situations recognition procedure during operation of oil wells. An approach was suggested to recognize technological situations associated with the operation of electrical centrifugal pumping units in oil production. The paper describes the methods for constructing models intended to recognize technological situations characterizing different types of failures of such electric centrifugal pumping (ECP) units. The models based on artificial neural networks, classification trees and support vector machines were considered as separate methods for constructing models for recognizing the technical state of ECP units in oil production. The paper presents the results of studying such methods in the tasks of assessing the technical state of several types of oil and gas production equipment. It is proposed to use sets of models enabling to integrate solutions of individual recognizers to improve situation recognition reliability. In the course of the research, tests were carried out on real operational data of ECP units. The research results showed that the use of such complex models will ensure a sufficiently high accuracy of recognition of technological situations. The proposed complex models provide higher stability of the results, which is confirmed by the results of statistical analysis of solutions obtained in the course of numerical experiments. Thus, it is shown that the proposed complex models for recognition of technological situations are an effective option to be used in object control systems during operation of oil producing wells.

Key words: technical state, electric centrifugal pump, recognition, classification, models, oils, technology

\section{INTRODUCTION}

The operation of process equipment in oil and gas production is one of the main processes that determine the combination of the most important characteristics in the implementation of oil and gas projects, including their efficiency and safety. The operating efficiency of oil and gas equipment, as well as safety, are the primary targets in the implementation of various control processes. This is true for the entire set of processes for the development of oil and gas fields, where borehole oil and gas production is one of the most important processes.

Currently, a significant number of oil producing wells are operated using electric centrifugal pumping (ECP) units $[1,2]$. In the conditions of implementing measures for rational operation ECP units can ensure high efficiency and safety at various stages of production [3]. In this regard, the problem of developing approaches and methods to improve the operational efficiency and safety of ECP units seems relevant with regard to the increasing requirements for efficiency, environmental friendliness and manageability of oil and gas projects in general $[3$, $4,5,6,7]$.

The introduction and use of advanced production planning and control systems at all levels: from strategic to tactical and operational is one of the main directions to improve the efficiency and safety of oil and gas equip- ment nowadays. Significant amounts of data accumulate at the operational level in conditions of the current use of modern control systems. These data characterize the processes directly related to the process equipment control and the acquisition of parametric information from it. The task of processing large volumes of such accumulated data that gains increasing relevance seems an important problem hindering the development of oil and gas production facilities in such conditions. Considering the availability of means and technologies for monitoring, measuring and transmitting data, the volumes of information taken from each operational facility, for example, from an ECP unit, increase, become available for processing and can be used to make decisions regarding the effective and safe operation of such equipment [8]. The volume of such information, combined with time restrictions and a significant amount of simultaneously acting operational factors is an important restriction that prevents the use of such information in a "manual", purely operator mode $[9,10]$.

To overcome such restrictions, decision support systems (DSSs) $[11,12]$ can be developed and implemented, for which there are a significant number of examples of successful application in many industries - energy [13, 14], medicine $[15,16,17]$, industrial production $[18,19,20$, 21] and others. 
Obviously, modern conditions characterizing the use of such DSSs require serious study of mathematical and algorithmic support [22]. In the most effective advanced DSS, along with the "classical" statistical models, data processing models and methods that refer to the category of datamining methods are widely used, including artificial neural networks $[23,24,25,26]$. These components of DSSs mainly determine the properties of the decisions generated by the DSS. Thus, the effectiveness of the formed decisions in terms of the production problem to be solved largely depends on the selection and elaboration of the corresponding components. The set of characteristics that determine the speed of obtaining decisions in the finished DSS, the ability to interpret the proposed decisions, the ability to adapt decision support models and their computational efficiency is also seen significant $[12,18]$.

It should also be noted that despite a fairly wide class of decision support tasks that are covered by already existing developments in terms of models and algorithms, there is no universal DSS capable of providing high efficiency in processes irrespective of industry specifics [24]. This requires elaboration of issues in terms of the development and research of advanced approaches to computational model support for DSS in each separate direction in the development of science and technology, and of the oil and gas industry in particular $[9,10]$.

In this regard, a relevant research area, discussed in this paper below, is to develop and study those methods and models that can be used as a DSS analytical computing core during the operation of oil and gas equipment, particularly, of ECP units, which are among the most demanded in the current production conditions. One of the most important areas of ensuring the operation of ECP units is considered - the recognition of technological situations of operation in terms of determining the state of a process unit and identifying the available defects of various kinds based on a set of diagnostic information.

The description of the task of recognizing technological situations in decision support tasks when analyzing the reliability of ECP units is given in Section "Recognition of Technological Situations". Section "Methods" describes the methods proposed to form decision support models for the problem in question. We propose a new method of forming an integrated model based on particular models obtained by the considered methods to improve the recognition efficiency of ECP units. Section "Experimental Part" provides information about the data set for testing and examining the effectiveness of the considered and proposed approaches. The results of the experimental studies carried out on the data sets obtained during the operation of actual ECP units are also provided. Section "Discussion" presents the analysis of the research results, and discusses the possibility of scaling up and improving the effectiveness of the proposed approach.

\section{RECOGNITION OF TECHNOLOGICAL SITUATIONS}

The task of technological situation recognition is one of the most important among those that must be solved when developing and using DSS, which are applied as part of advanced digital software for equipment operation. This is a complex problem covering a set of interrelated subtasks, each of which requires elaboration concerning identification of the most effective approaches and models used as basic analytical and computational decision support procedures. To determine technical state of the equipment being operated is one of the most characteristic tasks that must be addressed while operating the equipment. This task is fully consistent with the problem of rational use of process equipment, which would ensure the efficiency and safety of this process and oil production at a particular facility.

Obviously, the recognition of the technological situation associated with the assessment of the technical state of the equipment (ECPs used for oil production are considered within the framework of the paper) is an integral characteristic that enables, in the most general form, to determine the possibility or impossibility of operating the equipment. In a more detailed consideration, the results of the technical state assessment can be interpreted as technological situations related to the failure of individual items of equipment, their approach to the threshold critical state, or the emergence of factors causing complications in the equipment operation.

The efficiency of equipment operation is determined, among other things, by the need to select rational operating modes, which is fully true for the ECP units used in the operation of oil wells. Rationality is determined by the requirement to maintain the specified borehole operation modes, which corresponds to ensuring uninterrupted operation during specified time intervals and, if possible, extending the periods characterizing the prescribed steady-state flow rate of oil produced. Accordingly, the technical state recognition, the identification of ECP elements that are in a state close to the critical one, the selection of power saving operation modes in such cases will increase the awareness of the ECP operator when choosing such rational operating modes. Consequently, the validity and effectiveness of decisions made will increase, which is the goal of using DSS.

In terms of safety, the recognition of technological situations - in the option under consideration regarding the identification of technical state and gradual failures of ECP elements - is fully consistent with the task of technical diagnostics in ensuring the operational reliability of the equipment. On the one hand, decisions obtained with the help of models for identification of technical state can be used to substantiate a shutdown and the need for unscheduled maintenance and repair. On the other hand, the advising information can be a basis for making decisions on extending the period of equipment operation while ensuring the required level of safety and reliability, assessed by a set of highly accurate model decisions. 
Such a procedure can be taken as a principle for a rational condition-based maintenance model, which is generally considered to be more efficient and safe compared to the traditional model, which determines fixed maintenance intervals based on operating time. Moreover, in the case of strong decorrelation with the data of computational models identifying the "well-reservoir-ECP unit" system and the parametric data of the borehole oil production monitoring system, while forming solutions that maintain awareness of the normal working condition, the obtained models for identification of technical state can be considered as an informational research base for early signaling of complications during production, for example, the occurrence of drill string leakage intervals. This information can be used for the preventive preparation of special tools to eliminate complications (leakage), determine the parameters for their installation and select the modes for further borehole operation.

The results of such recognition, with sufficient reliability of the models used in combination with other computational and analytical models, can be integrated into the decision formation mechanism as a DSS component. Operating result of such a system being interpreted by an appropriate specialist can be a decision on the choice of the most rational modes of equipment (ECP units) operation, which consists in determining the set of parameters ensuring the required level of efficiency and safety. A decision to stop operation may be an alternative option in view of approaching the threshold condition of individual ECP elements and the need for planned shutdown of production, rather than an emergency one, as would be the case with a sudden failure of the corresponding elements of pumping units.

Currently, the volume of data included in the information base for recognizing technological situations, in particular, determining the technical state of equipment, is formed on the basis of the following two data sets:

- Data of parametric monitoring carried out by regular means of operation. Such data, on the one hand, are obtained by a standard set of sensors (probes) and provides the minimum required level of awareness, consistent with the need to solve standard object control tasks. From the viewpoint of information content, such data can be used in the task of determining the ECP technical state. However, their use in an explicit form requires the construction of rather complex hierarchical models for the evaluation of the resulting indicator of technical state. At the same time, the parametric part of such models is largely determined by a direct expert-analytical method, which makes it difficult to use in real-life tasks, when the variability of operating factors and ECP models leads to the individualization of each production facility. The sensitivity of models, constructed using only such data, to noise in measurement channels and random parameter fluctuations should also be mentioned. It seems reasonable to use such data in conjunction with the data of the second type - data from special monitoring systems.

- Data of special monitoring representing the implementation of one or several non-destructive testing methods appropriate to the operating conditions. Vibration monitoring at various points of the object under consideration - ECP (vibration-based diagnostics) is one of the most informative methods of technical diagnostics and monitoring. The selection of specific means of vibration diagnostics, the ways of their placement and data acquisition under ECP operating conditions is beyond the scope of the study presented in this paper. The data set transferred by the real ECP operator and described in Section "Experimental Part" was considered within the framework of the research.

\section{METHODS}

\section{Artificial neural networks}

Currently, artificial neural networks, along with other data mining technologies, are quite successfully used to solve a wide range of tasks that can be assigned to groups of problems of classification, forecasting and modeling [27, $28,29]$. Neural networks with multi-layer perceptron architecture are most commonly used (according to some sources, up to $80 \%$ of neural network applications). However, this type of neural networks is not devoid of a number of shortcomings, in some cases significantly limiting their effectiveness in solving a number of problems [30]. These disadvantages often include, for example, the impossibility of determining situations in solving classification problems, when a pattern that needs to be classified falls into an area of space for which training data is not available, etc. Radial basis function networks lack some of the drawbacks of multilayer perceptron networks, which, along with their fairly simple structure, made them one of the alternatives to multilayer perceptrons in solving classification problems [25, 26].

In most studies of radial basis function networks, Gaussian is used in the hidden-layer neurons, which ensures, as practice of using such networks shows, sufficient efficiency of the neural network:

$R_{t}(X)=\exp \left[\frac{-\|X-C\|^{2}}{\sigma_{i}^{2}}\right]$

where $X$ is an input vector, $C_{i}$ - a radial basis function center (a center of the appropriate cluster) used in the

$i$-th hidden-layer neuron of the network, $i=\overline{1, k}$, where $k$ is the number of neurons on the hidden layer of the network, and $\sigma_{i}$ is a parameter that determines the width of the radial basis function used in the $i$-th hidden-layer neuron of the network. The following parameters significantly affect a classification error, and as a result, should be effectively selected:

1. Placement of $C_{i}$ cluster centers corresponding to the neurons of a single hidden layer.

2. Determination of $\sigma_{i}$, the so-called "window width" 
the parameter which affects the width of the radial function used in the $i$-th hidden-layer neuron of the network.

Thus, learning a radial basis function network is reduced to restoring the density of each of the $P_{y}(x)$ classes using the selected fitting algorithm (for example, the Estimation Maximization (EM) algorithm). Learning results in the centers and variances of the components. Estimating the variances, we actually select the metrics with which the distances to the centers will be calculated. The EM-algorithm is considered quite an effective way to configure radial basis function networks. It greatly benefits in performance compared to gradient methods that are more often used to configure other types of neural networks.

\section{Decision trees}

Decision trees are a way of representing rules in a hierarchical, consistent structure. To make a decision on which class an object or situation belongs to, it is necessary to answer questions in the tree nodes, starting from its root, i.e. each node in the tree includes checking for a specific independent variable. Sometimes two independent variables are compared with each other in a tree node or some function is determined from one or several variables. If the result of the comparison in the tree node has the true value, the transition is made to the right node of the next level, if the result is false, the transition is made to the left node; then again the comparison associated with the corresponding node follows [31].

If a variable that is checked in a node takes categorical values, a branch descending from the tree node corresponds to each possible value. If a variable is a number, it is checked whether its value is greater or less than some constant. Sometimes the range of numerical values is divided into several intervals. In this case, a check is performed to see if the value falls into one of the intervals. The tree leaves correspond to the values of the dependent variable, i.e. the classes. An object belongs to a certain class if the values of its independent variables satisfy the conditions written in the tree nodes on the path from the root to the leaf corresponding to this class. Decision trees are easily converted into rules. The condition described in the tree nodes on the path to the leaf is written into the conditional part of such rules; the final part contains the value defined in the leaf [32].

The general principle of constructing decision trees based on the divide-and-conquer technique consists in recursive decomposition of a set of objects from a training set into subsets containing objects belonging to the same classes [33].

The most popular algorithms for constructing decision trees include ID3, C4.5, CART. These algorithms are implemented in the Statsoft Statistica applied data analysis package. Within the framework of the study, the C 4.5 algorithm was used, since its higher efficiency was shown on a set of test problems.

\section{Support vector machine}

The basic support vector machine is considered for the case of two classes. The object (hereinafter in the description of the method referred to as a point in space) is regarded as a dimensionality vector $p$. It is necessary to separate these points by a hyperplane of dimension $(p-1)$. Such a plane is called the classifier plane. Data can be separated using various hyperplanes. The best hyperplane is a hyperplane, in constructing which the separation and the difference between the 2 classes is maximum. The original algorithm for finding the optimal plane, the linear classifier, was proposed by Vapnik in 1953. However, in 1992, Bernhard E. Boser, Isabelle M. Guyon and Vladimir N. Vapnik proposed a method for creating a non-linear classifier using an arbitrary kernel function to find the planes of maximum difference [34].

A multiclass support vector machine is intended to classify objects in case of several (more than two) classes [35].

The dominant approach is the transition from the problem of classification into the sets of classes to the multiple task partition into 2 classes. Common methods for such a transition include [35]:

Construction of binary classifiers that distinguish:

- One class from the others (One-vs-All, OVA);

- One class from another (One-vs-One, OVO).

The classification of new objects using the OVA approach is carried out through a winner-takes-all strategy. The classifier, with the highest output function value, assigns a new object to a specific class (it is important that the function output can be calibrated to obtain comparable estimates).

For an OVO approach, classification is made using a max wins voting strategy, in which each classifier assigns an object to one of two classes.

\section{Proposed approach}

To increase the efficiency of the implementation of the decision formation stage in recognition tasks, a three-level method was proposed with the task decomposition in an implicit form. The steps of such a three-step method for forming an ensemble of classifiers are described below:

Stage 1. At this stage, a multitude of classifiers is formed independently. This stage is common to all collective approaches. In the general case, any available effective method for forming individual classifiers of the chosen type can be used. The number of resources available for use at this stage is determined proceeding from the general requirements for the problem solution time, the required accuracy and the available computational capabilities. Note that in the general case, any technologies used for classification and enabling to obtain a real or discrete output value that characterizes the belonging of an object described by an input vector to some class can be applied as classifiers at the first level. 
Stage 2. At the second stage, a set of second-level classifiers is formed independently of each other, coinciding with the number of classes in the considered problem. The inputs of classifiers of this level are the values obtained at the output of the first-level classifiers. In this case, for each $j$-th classifier of the second level $(j=1, m)$, training is carried out according to the following rule:

- The target value at the classifier output is 1 for all examples corresponding to the class with the number $j$;

- For all other examples, the target value at the classifier output is 0 .

Thus, at the second stage, the decomposition of the problem is performed - each second-level classifier forms a surface in space that "cuts off" objects of one class from objects belonging to any other classes. To solve this problem, the authors propose to use a method based on the application of hybrid genetic programming to combine the solutions of individual neural networks.

Stage 3. At the third stage, the decisions of the second-level classifiers are aggregated in order to develop a common solution - the values of the class for the input set. The selection of the rule to form a decision is a subject for further research. In the framework of the proposed approach, the authors used the following simple and, at the same time, obvious rule: the classified object belongs to the class for which the corresponding second-level classifier produced the maximum value of the output signal.

\section{EXPERIMENTAL PART}

\section{Dataset employed}

The dataset for analysis accumulated at the implementation site and similar facilities (in terms of functionality and composition of process equipment) in most modern systems is represented by fairly large amounts of data, which imposes high requirements for the efficiency and intensity of data use. The results of studying the effectiveness of the proposed methods on sets of data analysis tasks from 6 objects operating ECP at the oil fields in the Krasnoyarsk Krai are presented below. The data were obtained and formulated to set the problem of classifying ECP states arising from the ECP operation in oil production in various modes. The total number of records in the datasets combining the data about the use of special diagnostic modules (vibration diagnostics), parametric monitoring data and results of determining the ECP state at the time of maintenance and repair amounts to 540 units. The paper presents the data in an impersonal form, as they are commercial interest in their complete form.

\section{Method settings}

Artificial Neural Networks: The probabilistic PGNS method was used for designing the structure of neural networks. A hybrid genetic algorithm with parameters selected during the preliminary study was used to adjust the weights of neural networks.

Decision Tree Method: The standard C4.5 algorithm was applied.

Support Vector Machine: A software implementation of the method developed by the authors of the LIBSVM application software library was used. All parameters were set to the values recommended by the developers of the software library, and described by them as providing the best performance on average on the sets of tested tasks.

Proposed Approach: The first-level classifiers were formed by the methods discussed in the paper: artificial neural networks - radial basis function networks were used, the support vector machine was applied for the multiclass case and the decision tree method with C4.5 trees was used.

In the proposed three-stage method, classifiers obtained by genetic programming were used as second-level classifiers. The number of classifiers at this step is equivalent to the number of classes in a specific task. The number of generations in the genetic programming method for each classifier is 200 , the number of individuals per generation equals to 100 .

\section{Evaluation of the results obtained}

The study of the effectiveness of the considered approaches consisted in conducting a series of experiments and statistical evaluation of the results obtained.

When conducting the experiments, the following scheme was used: the total number of series of experiments for each method studied was 10 , in each series of experiments their evaluation was calculated by formula (2) for five times. Thus, the total number of launches conducted to evaluate the effectiveness of each approach was 50 .

At the same time, in each series of experiments, a new random division of the sample into the training and test sets was carried out in a ratio of $66 \%$ and $34 \%$ of the initial sample. To test the statistical significance of the results, they were verified by the ANOVA method at a significance level of 0.05 .

The estimate of the proportion of validly classified test sample examples, calculated using the formula below, was used as a criterion for the effectiveness of the algorithms:

$$
P=\frac{\text { Number of validly classified test sample examples }}{\text { Total number of test sample examples }} \cdot 100 \%
$$

\section{RESULTS}

The results of solving test problems are given in Table 1. The research results demonstrate that the proposed method implementing the ensemble (team) of classifiers is more effective than the majority of other studied approaches on the multitude of test sets used. In general, on average, the proposed approach is $10 \%$ more efficient than other methods in terms of the test functions. 
Table 1: The results of the study on the test set

\begin{tabular}{|l|c|}
\hline $\begin{array}{l}\text { The classification method } \\
\text { used to recognize the } \\
\text { ECP technical state }\end{array}$ & $\begin{array}{l}\text { Classification reliability } \\
\text { calculated by the formula } \\
2\end{array}$ \\
\hline Support vector machine & 86.1 \\
\hline Decision trees & 77.6 \\
\hline Artificial neural networks & 83.2 \\
\hline Proposed approach & 97.1 \\
\hline
\end{tabular}

\section{DISCUSSION}

The analysis of the results of studying the effectiveness of various methods for recognizing the ECP technical state based on the vibration diagnostics data and parametric monitoring data was conducted in the following areas:

- The overall effectiveness of the approaches, taking into account the complexity of the procedure for constructing models and the time spent on working out one solution under the operating conditions of the models obtained;

- Evaluation of the statistical parameters of the results obtained.

It should be noted that for all methods the same restriction was used on the amount of computational resources available to the method to get a solution for the purpose of obtaining correct results of numerical experiments.

The analysis of the problem solution progress makes it possible to conclude that the methods competing with the proposed approach have practically reached the threshold of their effectiveness, which was easily monitored by their insensitivity to the increase in computational resources allocated to form the solution. When using the proposed ensemble of classifiers, the efficiency of the ECP technical state recognition model was thus increased in terms of the "computational complexity-recognition accuracy" parameters. This fact is very significant in solving practical problems where a high degree of agreement between calculated and real data is very important.

In the course of the experimental study, it was shown that in the problem of technical state recognition, the considered methods of artificial neural networks, classification trees and the support vector machine give $86 \%$ accuracy on real data sets. With the help of the proposed approach, the recognition accuracy on the considered actual datasets was increased to $97 \%$. An expert analysis based on the opinions of specialists of enterprises operating the type of equipment in question (ECP) suggests that such an increase in the accuracy of problem solving (classification accuracy) is essential from the viewpoint of practical application of the methods under consideration.
With regard to the equality of resources allocated for constructing a model, which were estimated by using processor time on a computer applied for the research, the selection in favor of an approach that gives more accurate results seems to be obvious, i.e. the proposed method for recognizing the technical state based on a multilevel ensemble (classifier group) is preferable.

The statistical significance of the research results was confirmed for each approach by verification with the ANOVA method at a significance level of 0.05 . In general, the spread of the classification reliability estimate is explained by random sampling for construction of each classifier model, since in the course of the experiments fivefold cross-validation was applied to estimate the effectiveness. And each time the results were averaged by the results of the design and solution of the problem using five different classification models for each of the studied approaches.

\section{CONCLUSION}

The article deals with the task of developing and researching model formation methods for recognizing technological situations in oil production using ECP units. Within the framework of the presented part of the research, methods were considered for constructing classification models that can be used as part of the DSS computational analytical core employed in the operation of technological equipment for oil and gas production. The urgency of such a task was shown in the context of increasing amounts of information obtained during the operation of oil and gas equipment at the various levels of control. A significant amount of data, increasing requirements for the operational efficiency and safety of oil production equipment, in general, and ECP units, in particular, form a request for the use of effective decision support models in operating conditions. All these factors determine the need to develop and research relevant methods and models as a result of the application of such methods for the subsequent rational determination of operating modes.

The presented research is focused on the study of approaches for recognizing the ECP technical state based on diagnostic information obtained by vibration diagnostics sensors in combination with a set of operational parameters characteristic of ECP units. A separate direction of further research is the elaboration of the issues of technical implementation and optimization of the structural composition of the necessary diagnostic system to improve the quality of the ECP technical state recognition without additional tripping operations.

In the framework of the research presented in the paper, it was shown that methods related to data mining technologies can be used to solve the problem of recognizing the ECP technical state. Such methods as artificial neural networks, decision trees, and support vector machine were escribed and tested on the actual data. 
$\mathrm{n}$ the course of the experimental study, it was shown that in the technical state recognition problem, such methods give an accuracy of up to $86 \%$ on actual datasets. The analysis of statistical processing of the research results actualized the problem of increasing the accuracy of solving the technical state recognition problem when using such approaches.

The paper proposes a new method for forming the ECP technical state recognition models based on a set (ensemble) of models that enable to combine classification models constructed by any of the methods considered in the study. This method determines the formation of the second-level classification model, which generates the resulting solution based on the decisions of individual classifiers. The openness of this second-level model for the first-level classifiers seems important, which allows for the connection of universal analytical modules implementing various technologies of data analysis.

The obtained model for recognition of the ECP technological state during the operation of oil producing wells enabled to achieve the $97 \%$ accuracy in the technical state determination. The result obtained significantly exceeds the results for individual methods for constructing ECP state assessing models. In general, the achieved recognition efficiency is consistent with the accuracy requirements established for models and methods designed for promising DSS of process situation recognition during operation of oil and gas processing equipment.

Thus, the models obtained in the course of the study and the proposed approach to their formation can be used as a computational component of the DSS analytical part applied in evaluating the operational efficiency and safety of oil production equipment. A far-reaching possibility of integrating the solutions obtained with the models of computational and simulation modeling of the operation processes of production oil and gas wells seems important. This will provide a comprehensive analysis of technological situations during the operation of such wells, the identification of complications associated with both failures and technical state of equipment, in particular ECP, and complications caused by leakages in certain sections of wells, which requires the use of special equipment - packers.

Future research should be focused on the development and investigation of methods and models of analytical support for solving other problems of such DSS and integration of the proposed solutions in the form of a high-performance computing platform ensuring operation of oil production equipment.

\section{ACKNOWLEDGMENTS}

The work was completed with financial support from the Ministry of Education and Science of the Russian Federation within the framework of the Federal Target Program "Research and development in priority areas of scientific and technological complex of Russia for 2014 - 2020", action 1.3. Unique identifier of applied researches (project): RFMEFI57817X0236.

\section{REFERENCES}

1. Bortnikov, A.E., Valeev, M.D. (2011). Operation of electrical centrifugal pump units in flooded wells. Oil Industry, vol. 8, 61-63.

2. Coltharp, E.D. (1984). Subsurface electrical centrifugal pumps. JPT, Journal of Petroleum Technology, vol. 36, no. 4, 645-652, DOI: 10.2118/9982-PA

3. Gabdullin, R.F. (2002). Operation of wells equipped with centrifugal electrical pumps units under complicated conditions. Oil Industry, vol. 4, 62.

4. Koshtorev, N.I., Zayakin, V.I. (2001). Electrocentrifugal pumps with an articulated joint for oil production. Chemical and Petroleum Engineering, vol. 37, no. 9-10, 516-517, DOI: 10.1023/A:1013354901763

5. Mdee, O.J., Joseph, K., Kimambo, C.Z., Nielsen, T.K. (2016). Reversing centrifugal pumps as alternative to conventional turbines for micro hydropower: A review. Proceedings of the 5th IASTED International Conference on Power and Energy Systems, p. 373380.

6. Rodrigues, B.M., Cerqueira, A.A., Russo, C., Marques, M.R.C. (2010). Electroflocculation applied to the treatment of oil production wastewater. Periódico Tchê Química, vol. 7, no. 14, 7-15.

7. Cerqueira, A.A., Marques, M.R.C., Russo, C. (2010). Application of the technique of electroflocculation using alternate current in treatment of water production from oil industry. Periódico Tchê Química. vol. 7, no. $13,33-45$

8. Zyatikov, P.N., Kozyrev, I.N., Deeva, V.S. (2016). Operation effectiveness of wells by enhancing the electric-centrifugal pump. IOP Conference Series: Earth and Environmental Science, vol. 43, no. 1, 012078 , DOI: 10.1088/1755-1315/43/1/012078

9. Bukhtoyarov, V.V., Tynchenko, V.S., Petrovskiy, E.A., Tynchenko, V.V., Zhukov, V.G. (2018). Improvement of the methodology for determining reliability indicators of oil and gas equipment. International Review on Modelling and Simulations, vol. 11, no. 1, 37-50, DOI: 10.15866/iremos.v11i1.13994

10. Bukhtoyarov, V., Tynchenko, V., Petrovskiy, E., Bukhtoyarova, N., \& Zhukov, V. [2018]. investigation of methods for modeling petroleum refining facilities to improve the reliability of predictive decision models. Journal of Applied Engineering Science, 16(2), 246-253.

11. Bukharov, O.E., Bogolyubov, D.P. (2015). Development of a decision support system based on neural networks and a genetic algorithm. Expert Systems with Applications, vol. 42, no. 15-16, 6177-6183, DOI: 10.1016/j.eswa.2015.03.018 
12. Wu, X., Sun, H., Wu, Z., Miao, X. (2017). Modeling and evaluating of decision support system based on cost-sensitive multiclass classification algorithms. Advances in Intelligent Systems and Computing, vol. 541, 433-438.

13. Akdeniz, E., Bagriyanik, M. (2016). A knowledge based decision support algorithm for power transmission system vulnerability impact reduction. International Journal of Electrical Power and Energy Systems, vol. 78, 436-444, DOI: 10.1016/j. ijepes.2015.11.041

14. Alencar, T.R., Gramulia, J., Otobe, R.F., Asano, P.T.L. (2015). Decision support system based on genetic algorithms for optimizing the operation planning of hydrothermal power systems. 5th International Youth Conference on Energy, 7180815.

15. Yun, T., Yi, G.-S. (2008). Application of random forest algorithm for the decision support system of medical diagnosis with the selection of significant clinical test. Transactions of the Korean Institute of Electrical Engineers, vol. 57, no. 6, 1058-1062.

16. Yan, H., Ding, X., Peng, C., Xiao, S. (2004). Study on medical diagnosis decision support system for heart diseases based on hybrid genetic algorithm. Journal of Biomedical Engineering, vol. 21, no. 2, 302-305.

17. Yang, J., Wang, X., Dang, J. (2014). On the algorithm of the medical diagnostic decision support system under the mobile platform. Open Electrical and Electronic Engineering Journal, vol. 8, no. 1, 589593.

18. Capkovič, F. (1988). A decision support algorithm for flexible manufacturing systems control. Computers in Industry, vol. 10, no. 3, 165-170, DOI: 10.1016/01663615(88)90035-8

19. Murygin, A.V., Tynchenko, V.S., Laptenok, V.D., Emilova, O.A., Bocharov, A.N. (2017). Complex of automated equipment and technologies for waveguides soldering using induction heating. IOP Conference Series: Materials Science and Engineering, vol. 173, no. 1, 012023, DOI: 10.1088/1757899x/173/1/012023

20. Lachhab, M., Béler, C., Coudert, T. (2018). A riskbased approach applied to system engineering projects: A new learning based multi-criteria decision support tool based on an Ant Colony Algorithm. Engineering Applications of Artificial Intelligence, vol. 72, 310-326, DOI: 10.1016/j.engappai.2018.04.001

21. Jun Tan, C., Hanoun, S., Peng Lim, C. (2015). A multi-objective evolutionary algorithm-based decision support system: A case study on job-shop scheduling in manufacturing. 9th Annual IEEE International Systems Conference, p. 170-174.
22. Zimmermann, H.-J., Sebastian, H.-J. (1995). Intelligent system design support by fuzzy-multi-criteria decision making and/or evolutionary algorithms. IEEE International Conference on Fuzzy Systems, vol. 1, p. 367-374.

23. Milov, A.V., Tynchenko, V.S., Petrenko, V.E. (2019). Algorithmic and software to identify errors in measuring equipment during the formation of permanent joints. 2018 International Multi-Conference on Industrial Engineering and Modern Technologies, 8602515.

24. Peng, Y., Yang, X., Xu, W. (2018). Optimization research of decision support system based on data mining algorithm. Wireless Personal Communications, vol. 102, no. 4, 2913-2925, DOI: 10.1007/ s11277-018-5315-3

25. Tynchenko, V.S., Tynchenko, V.V., Bukhtoyarov, V.V., Tynchenko, S.V., Petrovskyi, E.A. (2016). The multi-objective optimization of complex objects neural network models. Indian Journal of Science and Technology, vol. 9, no. 29, 99467, DOI: 10.17485/ ijst/2016/v9i29/99467

26. Tynchenko, V.S., Petrovsky, E.A., Tynchenko, V.V. (2016). The parallel genetic algorithm for construction of technological objects neural network models. 2nd International Conference on Industrial Engineering, Applications and Manufacturing, 7911573.

27. Glorot, X., Bengio, Y. (2010). Understanding the difficulty of training deep feedforward neural networks. Proceedings of Machine Learning Research, no. 9, 249-256.

28. Ali, S.H., Ali, A.H. (2019). Crude oil price prediction based on soft computing model: Case study of Iraq. Journal of Southwest Jiaotong University, vol. 54, no. 4, DOI: 10.35741/issn.0258-2724.54.4.36

29. Mahmood, M., Al-Kubaisy, W.J., Al-Khateeb, B. (2019). Using artificial neural network for multimedia information retrieval. Journal of Southwest Jiaotong University, vol. 54, no. 3, DOI: 10.35741/issn.02582724.54.3.19

30. Zhang, G., Patuwo, B.E., Hu, M.Y. (1998). Forecasting with artificial neural networks: The state of the art. International Journal of Forecasting, vol. 14, no. 1, 35-62, DOI: 10.1016/S0169-2070(97)00044-7

31. Quinlan, J.R. (1986). Induction of decision trees. Machine Learning, vol. 1, no. 1, 81-106, DOI: 10.1023/A:1022643204877

32. Geurts, P., Ernst, D., Wehenkel, L. (2006). Extremely randomized trees. Machine Learning, vol. 63, no. 1, 3-42, DOI: 10.1007/s10994-006-6226-1 
33. Quinlan, J.R. (1987). Simplifying decision trees. International Journal of Man-Machine Studies, vol. 27, no. 3, 221-234, DOI: 10.1016/S00207373(87)80053-6

34. Boser, B.E., Guyon, I.M., Vapnik, V.N. (1992). A training algorithm for optimal margin classifiers. Proceedings of the 5th Annual ACM Workshop on Computational Learning Theory, p. 144-152.
35. Hsu, C.-W., Lin, C.-J. (2002). A comparison of methods for multiclass support vector machines. IEEE Transactions on Neural Networks, vol. 13, no. 2, 415-425, DOI: 10.1109/72.991427

Paper submitted: 22.08.2019.

Paper accepted: 22.10.2019.

This is an open access article distributed under the CC $B Y-N C-N D 4.0$ terms and conditions. 